Les points $\xi$ arec $f(\xi)>f(\alpha)$, tout comme ceux avec $f(\xi)<f(\alpha)$, constituant un ensemble partout dense, on a en vertu de la continuité de $E(x)$

$$
E(x) \equiv \frac{x-\alpha}{2}
$$

Mais c'est incompatible avec le théorème suivant de Jacobsthal et Knopp [3]: si pour un ensemble $E$ et pour tout intervalle $I$ on a

$$
\frac{|E I|}{|I|}=\text { const. }
$$

ce rapport est égal à 0 ou à 1 (c'est incompatible aussi avec le théorème de Lebesgue sur les points de densité).

\title{
Bibliographie.
}

[1] G. Hamel, Math. Ann. 60 (1905), p. 460.

[2] A. L. Cauchy, Analyse algebrique, Paris 1821, Oeuvres IIo série, t. III, pp. 98-105. p. 121.

[3] Jacobsthal et Knopp, Sitzungsb. d. Berl. Math. Ges. 14 (1915)

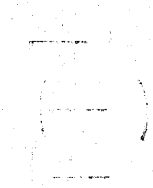

\section{Cancellation laws in the arithmetic of cardinals.}

By

\author{
Alfred Tarski (Berkeley, California, U.S.A.).
}

Dedicated to Frofessor Waclaw Sierpinsti in celebration of his forty years as teacher and scholar.

In this paper I should like to outline a proof of the following two cancellation laws for finite multiples of cardinal numbers:

I. Given a natural number $m \neq 0$ and two arbitrary cardinals, $\mathfrak{p}$ and $\mathfrak{q}$, if $m \cdot \mathfrak{p}=m \cdot \mathfrak{q}$, then $\mathfrak{p}=\mathfrak{q}$.

II. Given a natural number $m \neq 0$ and two arbitrary cardinals, $\mathfrak{p}$ and $\mathfrak{q}$, if $m \cdot \mathfrak{p} \leqslant m \cdot \mathfrak{q}$, then $\mathfrak{p} \leqslant \mathfrak{q}$.

Both $I$ and II belong to those theorems of the arithmetic of cardinals which ean be proved in an effetive way, without the help of the axiom of choice, and which, as a consequence of the structure of their proofs, can be extended to a comprehensive class of abstract algebraic systems referred to as cardinal algebras ${ }^{1}$ ).

It may be noticed that the two theorems discuissed can be derived as immediate corollaries from the familiar theorem of the arithmetic of cardinals by which a finite muliple $m \cdot p(m \neq 0)$ of an infinite cardinal $p$ always equals $p$. The proof of this last theorem, however, involves essentially the well-ordering principle; hence the possibility of deriving $I$ and $I I$ from it will be disregarded in the present discussion, for we shall be interested only in arguments which aroid any explicit or implicit application of the axiom of choice.

x) The algebraic aspect of the problem will not be discussed in this paper. See in this figures in brackets referring to the bibliography at the end of the paper). It may be mentioned that with one exception - the results stated in this paper in a formal way (i. e., with one exception - the results stated in this paper in a formal way (1. e., theorems and corollaries 1-14) can be extended to arbitrary cardinal algebras, this one exception (Theorem 7), see Footnote 16 below. 
An effective proof of $\mathrm{I}$ and II is by no means simple. As: a matter of fact, no such proof of Theorem II and no detailed proof of Theorem I have been available in the literature until recently.

Theorem $\mathbf{I}$ for $m=2$ was first proved by F. Bernstein; for the general case Bernstein gave only a rough outline of a proof, the understanding of which presents some difficulties 2). Another, very elegant proof of $I$ in the case $m=2$ was published later by W. Sierpiński ${ }^{3}$ ); and a proof in the general case was found, but not published, by the late A: Lindenbaum ${ }^{4}$ ). Theorem $\mathrm{IT}$ - from which I can obviously be derived by means of the Cantor-Bernstein equivalence theorem - was first obtained for $m=2$ by myself, and then extended to the general case by Lindenbaum; the proofs, however, were not published $\left.{ }^{4}\right)$. Recently Sierpiński has published a proof of II for $m=2^{5}$ ).

A few year ago I found two different proofs of Theorem II. (and hence also, indirectly, of Theorem I). One of these proofs can be obtained by analyzing the proof of the corresponding theorem applying to arbitrary cardinal algebras; the proof of this algebraic theorem is published elsewhere $\left.{ }^{6}\right)$. The second proof is just the one which I should like to present in this paper. It is in " a sense an extension of the original proof given by me for $m=2$, and is undoubtedly related to Lindenbaum's proof for the general case. Unfortunately, I am not in a position to state how close this relation is. The only facts concerning Lindenbaum's proof which I clearly remember are the following: the proof was based on a weaker though related result previously obtained by me, which will be given below as Theorem 6; the idea used in an important part of the proof was. rather similar to the one used by Sierpiniski in the abovementioned proof of Theorem I for $m=2$. Both these facts apply as well to the proof I am going to outline. On the other hand, my proof will be based upon a lemma given as Theorem 7, which seems.

2) See Bernstein [1], pp. $122 \mathrm{ff}$.

3) See Sierpiński [2].

4) See Lindenbaum-Tarski [1], p. 305.

s) See Sierpiński [3].

6) In fact, in Tarski [2], pp. $30 \mathrm{ff}$. The algebraic proof referred to involves a restricted application of the axiom of choice. However, when specializing the proof and applying it, not to abstract cardinal algebras, but specifically to. cardinal numbers, one easily notices that the use of the axiom of choice can be avoided. Cf. in this connection op. cit., pp. 239 ff., as well as Tarski [1], §2. to be a new result and which may present some interest in itself; it is, however, by no means excluded that the proof of this lemma could have been easily obtained by analyzing Lindenbaum's argument.

It should be pointed out that - as opposed to Theorems I and II - the general cancellation laws for products do not hold in the arithmetic of cardinals: $\mathfrak{n} \cdot \mathfrak{p}=\mathfrak{r} \cdot \mathfrak{q}$ does not always imply $\mathfrak{p}=\mathfrak{q}$, nor does $\mathfrak{n} \cdot \mathfrak{p} \leqslant \mathfrak{n} \cdot \mathfrak{q}$ always imply $\mathfrak{p} \leqslant \mathfrak{q}$ (even if $\mathfrak{n} \neq 0$ ). The same remark applies to the general cancellation laws for sums; however, we shall come across certain particular cases of these laws (Theorems 3 and Corollaries 4 and 5 below) which hold in the arithmetic of cardinals and can even be obtained without the help of the axiom of choice.

We shall use in this paper various familiar set-theoretical notions and symbols ${ }^{7}$ ). In particular, given a function $f$, we shall denote by $D(f)$ the domain - the set of argument values - of $f$. If $f$ is biunique, then the domain of its inverse, $D\left(f^{-1}\right)$, obviously coincides with the range (counter-domain) - the set of function values - of $f$. The composition of two functions $f$ and $g$ will be denoted as usual by $f g$. The composition is defined even if the range of $g$ is not included in the domain of $f$; in this case, however, $D(f g)$ is a proper part of $D(g)$.

Given a set $A$ and a function $f$, we shall denote by $f(A)$ the $f$-image of $A$, i. e., the set of all function values $f(x)$ correlated with those elements $x \in A$ which are in $D(f)$; in symbols

$$
f(A)=\underset{f(x)}{E}[x \in A] .
$$

(Thus, the functional notation is used ambiguously, but I believe: that no confusion will arise from this ambiguity). The set $A$ does not have to be included in the domain of $f$; the set $f(A)$ is always included in the range of $f$. We shall apply various elementary properties of images, e. g., the following ones:

$f\left(\sum_{i} A_{i}\right)=\sum_{i} f\left(A_{i}\right)$ for every system of sets $A_{i}$

$f\left(\prod_{i} A_{i}\right)=\prod_{i} f\left(A_{i}\right)$ for every non-empty system of sets $A_{i}$;

$f(\stackrel{A}{A}-B)=f(A)-f(B)$ for any sets $A$ and $B$;

$f^{-1} f(A)=A \cdot D(f)$ for every set $A$; $f\left(A \cdot f^{-1}(B)\right)=f(A) \cdot B$ for any sets $A$ and $B$.

7) For set-theoretical notions and results referred to or applied in thise paper consult, for instance, Sierpiński [1]. 
The first of the above formulas holds for any arbitrary function $f$; the remaining ones are based upon the assumption that $f$ is biunique - though the last two can also be applied to arbitrary functions, under an appropriate definition of $f^{-1}(A)$.

The Roman letters $i, j, \ldots, m, n, \ldots$ will represent arbitrary natural numbers, and the German letters $\mathfrak{n}, \mathfrak{p}, \ldots$ arbitrary cardinals. The notion of the sum $\mathfrak{n}+\mathfrak{p}$ of two cardinals $\pi$ and $\mathfrak{p}$ is of course assumed to be known. In terms of this notion, the relations $\geqslant$ and $\leqslant$ between cardinals, the sum $\sum_{i=1}^{m} \mathfrak{n}_{i}$ of a finite sequence $\mathfrak{t}_{1}, \ldots, \mathfrak{t}_{m}$ of cardinals, and the $m^{\text {th }}$ multiple $m \cdot \mathfrak{n}$ of a cardinal $\mathfrak{n}$ can be defined in a familiar way; e. $g$., $\mathfrak{n} \geqslant \mathfrak{p}$ means that there is a cardinal $\mathfrak{q}$ for which $\mathfrak{n}=\mathrm{p}+\mathfrak{q}$. We shall freely apply various elementary theorems concerning these notion whose proofs do not involve the axiom of choice - such as the commutative and associative laws for addition. Moreover, in the proofs of our fundamental theorems we shall use (directly or indirectly) several results of a less elementary character stated below as theorems and corollaries 1-7.

Theorem 1. If $\mathfrak{n} \leqslant \mathfrak{p}$ and $\mathfrak{n} \geqslant \mathfrak{p}$, then $\mathfrak{n}=\mathfrak{p}$.

This is, of course, the well-known Cantor-Bernstein equivalence theorem.

Theorem 2. If $\mathfrak{n}+\mathfrak{p}=\mathfrak{n}+\mathfrak{q}$, then there are cardinats $\mathfrak{n}^{\prime}, \mathfrak{p}^{\prime}$, and $\mathfrak{q}^{\prime}$ such that

$$
\mathfrak{n}=\mathfrak{n}+\mathfrak{p}^{\prime}=\mathfrak{n}+\mathfrak{q}^{\prime}, \quad \mathfrak{p}=\mathfrak{n}^{\prime}+\mathfrak{p}^{\prime} \text {, and } \mathfrak{q}=\mathfrak{n}^{\prime}+\mathfrak{q}^{\prime} \text {. }
$$

The proof of this theorem is published elsewhere ${ }^{8}$ ).

Theorem 2 replaces to some extent the general cancellation laws for sums, which - as was mentioned above - do not apply to arbitrary cardinals. Its content can be described as follows: if $\mathfrak{n}+\mathfrak{p}=\mathfrak{n}+\mathfrak{q}$, then $\mathfrak{p}$ and $q$ are equal up to certain cardinals $\mathfrak{p}^{\prime}$ and $\mathfrak{q}^{\prime}$ which are „absorbed” by $\mathfrak{n}$ (in the sense that $\mathfrak{n}=\mathfrak{n}+\mathfrak{p}^{\prime}=\mathfrak{n}+\mathfrak{q}^{\prime}$ ). Hence a related result for the inequality $\mathfrak{n}+\mathfrak{p} \leqslant \mathfrak{n}+\mathfrak{q}$ can easily be derived, but will not be formulated here $\%$ ).

8) See Tarski [2], pp. $18 \mathrm{f}$. (keeping in mind the remarks made in Footnote 6), or Tarski [1], \& 1 (Lemma A in the proof of Theorem 2).

) See Tarski [2], p. 19, Corollary 2.7 .
Theorem 3. If $k \cdot \mathfrak{n}+\mathfrak{p} \leqslant(k+1) \cdot \mathfrak{n}+\mathfrak{q}$, then $\left.\mathfrak{p} \leqslant \mathfrak{n}+\mathfrak{q}^{10}\right)$.

Proof: We apply induction with respect to $k$. The theorem being obvious for $k=0$, we suppose that it holds for a given $k=m$; we want to show that it also holds for $k=m+1$. Thus, assume

$$
(m+1) \cdot \mathfrak{n}+\mathfrak{p} \leqslant(m+2) \cdot \mathfrak{n}+q \text {. }
$$

Hence, for some cardinal $r$,

and therefore

$$
(m+1) \cdot \mathfrak{r}+\mathfrak{p}+\mathfrak{r}=(m+2) \cdot \mathfrak{r}+\mathfrak{q},
$$

$$
\mathfrak{n}+(m \cdot \mathfrak{n}+\mathfrak{p}+\mathfrak{r})=\mathfrak{n}+[(m+1) \cdot \mathfrak{n}+\mathfrak{q}] .
$$

By now applying Theorem 2, with $\mathfrak{p}$ and $q$ replaced by $m \cdot n+p+r$ and $(m+1) \cdot n+q$, respectively, we conclude from (1) that there are cardinals $\mathfrak{r}^{\prime}, \mathfrak{p}^{\prime}$ and $\mathfrak{q}^{\prime}$ such that

$$
\begin{gathered}
\mathfrak{n}=\mathfrak{n}+\mathfrak{p}^{\prime}=\mathfrak{n}+\mathfrak{q}^{\prime}, \\
m \cdot \mathfrak{n}+\mathfrak{p}+\mathfrak{r}=\mathfrak{n}^{\prime}+\mathfrak{p}^{\prime},
\end{gathered}
$$

and

$$
\begin{aligned}
\text { By (4) we have } \begin{aligned}
&(m+1) \cdot \mathfrak{n}+\mathfrak{q}=\mathfrak{n}^{\prime}+\mathfrak{q}^{\prime} . \\
&(m+1) \cdot \mathfrak{n}+\mathfrak{q}+\mathfrak{p}^{\prime}=\mathfrak{n}^{\prime}+\mathfrak{q}^{\prime}+\mathfrak{p}^{\prime} ;
\end{aligned}
\end{aligned}
$$

hence, by (2) and (3),

so that finally

$$
(m+1) \cdot \mathfrak{n}+q=q^{\prime}+m \cdot \mathfrak{n}+\mathfrak{p}+\mathfrak{r},
$$

$$
m \cdot \mathfrak{n}+\mathfrak{p} \leqslant(m+1) \cdot \mathfrak{n}+q \text {. }
$$

The inductive premise, when applied to the latter formula, gives at once

$$
\mathfrak{p} \leqslant \mathfrak{n}+\mathfrak{q} \text {. }
$$

Thus, our theorem holds for $k=m+1$, and hence also for an arbitrary natural $k$.

Corollary 4. If $k \cdot \mathfrak{n}+\mathrm{p} \leqslant k \cdot \mathfrak{n}+\mathfrak{q}$, then $\mathfrak{n}+\mathfrak{p} \leqslant \mathfrak{n}+q$.

Proof: For $k_{k}=0$ the corollary is obvious, and for $k>0$ it follows from Theorem 3 (with $k$ replaced by $k-1$, and $\mathfrak{p}$ by $\mathfrak{n}+\mathfrak{p}$ ).

10) Theorem 3 was stated in Lindenbaum-Tarski [1], p. 303. A particular case of this theorem $(k=1, q=0$, equality instead of inequality in the hypothesis) had been previously established in Bernstein [1]; the theorem in its generail form can be rather easily derived from this particular case.

Fundamenta Mathematicae. T. Xxxv1. 
While in Theorem 3 we cannot replace the symbol $\leqslant$ by $=$, we can do this in Corollary 4 (which is a more symmetric, though weaker, form of Theorem 3):

Corollary 5. If $k \cdot \mathfrak{n}+\mathfrak{p}=k \cdot \mathfrak{n}+\mathfrak{q}$, then $\mathfrak{n}+\mathfrak{p}=\mathfrak{n}+\mathfrak{q}$.

Proof: By Theorem 1 and Corollary 4.

From Theorem 2 (with the help of Theorem 1) we can derive still another consequence which - like Theorem 3 and Corollaries 4 . and 5 -is related to the cancellation laws for sums. In fact, we can easily show that:

For every cardinal $n$ the following three conditions are equivalent:

(i) $\mathfrak{n} \neq \mathfrak{n}+1$ (i. e., $\mathfrak{n}$ is finite in the sense of Dedekind);

(ii) $\mathfrak{n}+\mathfrak{p}=\mathfrak{n}+\mathfrak{q}$ aluays implies $\mathfrak{p}=\mathfrak{q}$;

(iii) $\mathfrak{n}+\mathfrak{p} \leqslant \mathfrak{n}+\mathfrak{q}$ always implies $\mathfrak{p} \leqslant \mathfrak{q}^{11}$ ).

Theorem 6. If $m \neq 0, m \cdot \mathfrak{p} \leqslant m \cdot \mathfrak{q}$, and $p \geqslant q$, then $p=q$.

Proof: By hypothesis there are cardinals $\mathfrak{r}$ and $\mathfrak{s}$ such that

and

$$
m \cdot \mathfrak{p}+\mathfrak{r}=m \cdot \mathfrak{q}
$$

From (1) and (2) we obtain

$$
m \cdot \mathfrak{q}+(m \cdot \mathfrak{s}+\mathfrak{r})=m \cdot \mathfrak{q}+0,
$$

and consequently, by Corollary 5 ,

$$
\mathfrak{q}+m \cdot \mathfrak{s}+\mathfrak{x}=\mathfrak{q}+0=\mathfrak{q} .
$$

Since $m \neq 0$, formulas (2) and (3) imply

$$
p \leqslant q .
$$

Hence, and from the last part of the hypothesis, the conclusion follows at once by Theorem 1.

If we define the $<$ relation as one which holds between two cardinals $\mathfrak{p}$ and $\mathfrak{q}$ if, and only if, $\mathfrak{p} \leqslant \mathfrak{q}$ and $\mathfrak{p} \neq \mathfrak{q}$, then we can give Theorem 6 the following equivalent form:

$$
\text { If } m \neq 0 \text { and } \mathfrak{p}<\mathfrak{q} \text {, then } m \cdot \mathfrak{p}<m \cdot \mathfrak{q}^{12} \text { ). }
$$

The proof of the equivalence is based upon Theorem 1 .

11) Compare Tarski [2], p. 57.

12) In this form Theorem 6 is stated in Lindenbaum-Tarski [1], p. 305.
It could seem at first glance that the cancellation laws for finite multiples are direct consequences of Theorem 6. This is, however, not the case since the trichotomy law (by which either $\mathfrak{p} \leqslant \mathfrak{q}$ or $\mathfrak{p} \geqslant \mathfrak{q}$ for any two cardinals $\mathfrak{p}$ and $\mathfrak{q}$ ) cannot be proved without the help of the axiom of choice, and hence is not available for our purposes. The following theorem will make up for the lack of the trichotomy law in our further diseussion:

Theorem \%. Let $k \geqslant 2$. Let $m_{1}, \ldots, m_{k}$ and $n_{1}, \ldots, n_{k}$ be two finite sequences of natural numbers, and $\mathfrak{p}_{1}, \ldots, p_{k}$ be a.finite sequence of cardinals, such that

$$
\sum_{i=1}^{k}\left(m_{i} \cdot p_{i}\right)=\sum_{i=1}^{k}\left(n_{i} \cdot \mathfrak{p}_{i}\right)
$$

Then there are two sequences of cardinals, $\mathfrak{r}_{1}, \ldots, \mathfrak{r}_{k}$ and $\mathfrak{s}_{1}, \ldots, \mathfrak{s}_{k}$, satisfying the following conditions:
(i) $\mathfrak{p}_{i}=\mathrm{r}_{i}+\mathfrak{s}_{i}$ for $i=1,2, \ldots, k$;
(ii) $\sum_{i=1}^{k}\left(m_{i} \cdot \mathfrak{r}_{i}\right)=\sum_{i=1}^{k}\left(n_{t} \cdot \mathfrak{r}_{i}\right)$ and $\mathfrak{r}_{1} \geqslant \mathfrak{r}_{2}$;
(iii) $\sum_{i=1}^{k}\left(m_{i} \cdot \mathfrak{s}_{i}\right)=\sum_{i=1}^{k}\left(n_{i} \cdot \mathfrak{s}_{i}\right)$ and $\mathfrak{s}_{1} \leqslant \mathfrak{s}_{2}$.

Proof: Without loss of generality we can elearly assume that $m_{i}+n_{i} \neq 0$ for $i=1,2, \ldots, k$.

By hypothesis, there is a set $P$ such that

$$
\overline{\bar{P}}=\sum_{i=1}^{k}\left(m_{i} \cdot \mathfrak{p}_{i}\right)=\sum_{i=1}^{k}\left(n_{i} \cdot \mathfrak{p}_{i}\right)
$$

Hence there exists a system of sets $P_{i, j}$ with $1 \leqslant i \leqslant k$ and $1 \leqslant j \leqslant m_{i}+n_{i}$, satisfying the following conditions:

$$
P=\sum_{i=1}^{k} \sum_{j=1}^{m_{i}} P_{i, j}=\sum_{i=1}^{k} \sum_{j=1}^{n_{i}} P_{i, m_{i}+f}
$$

(3) $P_{i, j} \cdot P_{i^{\prime}, j^{\prime}}=0$ if the ordered couples $(i, j)$ and $\left(i^{\prime}, j^{\prime}\right)$ are not identical, $1 \leqslant i \leqslant i^{\prime} \leqslant k$, and either $1 \leqslant j \leqslant m_{i}$ and $1 \leqslant j^{\prime} \leqslant m^{\prime}$ or else $1 \leqslant j-m_{i} \leqslant n_{i}$ and $1 \leqslant j^{\prime}-m_{i^{\prime}} \leqslant n_{i^{\prime}}$

(4) $\overline{\overline{P_{t, j}}}=\mathfrak{P}_{i}$ for $1 \leqslant i \leqslant k$ and $1 \leqslant j \leqslant m_{i}+n_{i}$. 
By (4), any two sets $P_{i, 1}$ and $P_{i, j}$ have the same power. Consequently, there exists a system of functions $f_{l, j}$ (where $1 \leqslant i \leqslant k$ and $1 \leqslant j \leqslant m_{i}+n_{i}$ ) with the following properties:

(5) each of the functions $f_{l, f}$ is 'biunique;

$$
D\left(f_{i, j}\right)=P_{i, 1} \text { and } D\left(f_{i, j}^{-1}\right)=P_{t, j} .
$$

We can also assume that

$$
f_{i, 1}(x)=x \text { for } \quad x \in P_{l, 1} .
$$

Consider all the functions $g$ obtained by iterated composition from the functions $f_{i, j}$ and their inverses $f_{i, j}^{-1}$. Since the set of all these functions $g$ is at most denumerable and not empty, we can arrange its elements in an infinite sequence $g_{1}, g_{2}, \ldots, g_{m}, \ldots$ Thus,

(8) every function $g_{m}$ is a composition of finitely many functions $f_{i, j}$ and $f_{i, f}^{-1}$; and, conversely, every such composition is one of the functions $g_{m}$.

From $(2),(5),(6)$, and (8) we conclude:

(9) all the functions $g_{m}(m=1,2, \ldots)$ are biunique;

(10) $D\left(g_{m}\right) \subset P$ and $D\left(g_{m}^{-1}\right) \subset P$;

(11) for any $i$ and $j, 1 \leqslant i \leqslant l$ and $1 \leqslant j \leqslant m_{i}+n_{i}$, there is a positive integer $m$ such that $f_{i, j}=g_{m}$;

(12) for every positive integer $m$ there is a positive integer $n$ such that $g_{m}^{-1}=g_{n}$

(13) for any positive integers $m$ and $n$ there is a positive integer $p$ such that $g_{m} g_{n}=g_{p}$.

We now define recursively an infinite sequence of sets $Q_{1}, Q_{2}, \ldots, Q_{m}, \ldots$ by setting

and

$$
Q_{1}=P_{1,1} \cdot g_{1}^{-1}\left(P_{2,1}\right)
$$

(15) We also put

$$
Q_{m+1}=\left(P_{1,1}-\sum_{i=1}^{m} Q_{i}\right) \cdot g_{m+1}^{-1}\left(P_{2,1}-\sum_{i=1}^{m} g_{i}\left(Q_{t}\right)\right) \quad \text { for } m=1,2, \ldots
$$

$$
R=\sum_{n=1}^{\infty} g_{n}\left(\ddot{P}_{1,1}-\sum_{m=1}^{\infty} Q_{m}\right) \quad \text { and } \quad S=P-R
$$

(17) $R_{i, j}=P_{i, j} \cdot R$ and $S_{i, j}=P_{i, j} \cdot S$ for $1 \leqslant i \leqslant k$ and $1 \leqslant j \leqslant m_{i}+n_{i}$;

$$
\mathfrak{r}_{i}=\overline{\overline{R_{i, 1}}} \text { and } \mathfrak{s}_{i}=\overline{\overline{S_{i, 1}}} \text { for } i=1,2, \ldots, k \text {. }
$$

We are going to show that the cardinals $\mathfrak{x}_{t}$ and $\mathfrak{s}_{i}$ defined in (18) satisfy all conditions stated in the conclusion of our theorem.

By (10) and (16) we have

$$
P=R+S \text { and } R \cdot S=0 \text {; }
$$

hence by (2) and (17)

$$
P_{i, j}=R_{i, j}+S_{i, j} \text { and } R_{i, j} \cdot S_{i, j}=0
$$

(where $1 \leqslant i \leqslant k$ and $1 \leqslant j \leqslant m_{i}+n_{i}$ ). Consequently, by (18),

$$
p_{i}=r_{i}+s_{i} \text { for } i=1,2, \ldots, k \text {. }
$$

From (13) and (16) we conclude that, for every positive integer $m$,

hence, by (12),

$$
\begin{aligned}
& g_{m}(R) \subset R \\
& g_{m}^{-1}(R) \subset R .
\end{aligned}
$$

As is easily seen, the two inclusions just stated give

$$
g_{m}(R)=R \cdot D\left(g_{m}^{-1}\right) \text { for } m=1,2,3, \ldots
$$

Furthermore, we obtain from (9), (10), (16), and (21)

and hence

$$
g_{m}(S)=g_{m}(P)-g_{m}(R)=P \cdot D\left(g_{m}^{-1}\right)-R ;
$$

$$
g_{m}(S)=S \cdot D\left(g_{m}^{-1}\right)
$$

By (5), (6), (11), (17), and (21) we have :

as well as

$$
f_{i, j}\left(R_{i, 1}\right)=f_{i, j}\left(P_{i, 1}\right) \cdot f_{i, j}(R)=P_{i, j} \cdot R=\dot{R}_{i, j}
$$

$$
R_{i, 1} \subset D\left(f_{i, s}\right) ;
$$

and we conclude that the sets $R_{i, 1}$ and $R_{i, j}$ with $1 \leqslant i \leqslant k$ and $1 \leqslant j \leqslant m_{i}+n_{i}$ have the same power. By using (22) instead of (21), we obtain the same conclusion for $S_{i, 1}$ and $S_{i, d}$. Therefore, by (18),

(23) $\overline{\overline{R_{i, j}}}=x_{i}$ and $\overline{\overline{S_{i, j}}}=s_{i}$ for $1 \leqslant i \leqslant k$ and $1 \leqslant j \leqslant m_{i}+n_{i}$.

We also see from (17) and (19) that conditions (2) and (3) are satisfied if we replace in them the sets $P$ and $P_{i, j}$ by $R$ and $R_{i, j}$, or $S$ and $S_{i, j}$, respectively. Hence, in view of (23), formula (1) will also hold if $P$ and $p_{i}$ are replaced by $R$ and $\mathfrak{r}_{i}$, or $S$ and $\mathfrak{s}_{i}$. Thus,

$$
\sum_{i=1}^{k}\left(m_{i} \cdot r_{i}\right)=\sum_{i=1}^{k}\left(n_{i} \cdot r_{i}\right) \text { and } \sum_{i=1}^{k}\left(m_{i} \cdot s_{i}\right)=\sum_{i=1}^{k}\left(n_{i} \cdot \varsigma_{l}\right) \text {. }
$$


It remains to be shown that the cardinals $\mathfrak{r}_{1}, \mathfrak{r}_{2}, \mathfrak{s}_{1}$, and $\mathfrak{s}_{2}$ satisfy the inequalities stated in conditions (ii) and (iii) of the conclusion. In this part of the proof we make an essential use of formulas (14) and (15) defining the sets $Q_{m}$. We first notice that, as a consequence of these formulas, we have for every positive integer $m$ (thus also for $m=1$ )

$$
Q_{m}=\left(P_{1,1}-\sum_{t=1}^{m-1} Q_{t}\right) \cdot g_{m}^{-1}\left(P_{2,1}-\sum_{i=1}^{m-1} g_{i}\left(Q_{i}\right)\right)
$$

and hence, with the help of (9),

$$
g_{m}\left(Q_{m}\right)=\left(P_{2,1}-\sum_{i=1}^{m-1} g_{i}\left(Q_{i}\right)\right) \cdot g_{m}\left(P_{1,1}-\sum_{i=1}^{m-1} Q_{i}\right) \text {. }
$$

From (25) and (26) we obtain

and

$$
Q_{m} \subset D\left(g_{m}\right) \text { for } 1 \leqslant m<\infty \text {. }
$$

$$
Q_{m} \cdot Q_{n}=0=g_{m}\left(Q_{m}\right) \cdot g_{n}\left(Q_{n}\right) \text { for } 1 \leqslant m<n<\infty .
$$

Hence, by setting

$$
h(x)=g_{m}(x) \text { for } x \in Q_{m}(m=1,2, \ldots),
$$

we obtain a biunique function $h$ which maps respectively the sets

onto the sets

$$
\sum_{m=1}^{\infty} Q_{m}, \sum_{m=1}^{\infty}\left(Q_{m} \cdot R_{1,1}\right), \text { and } \sum_{m=1}^{\infty}\left(Q_{m} \cdot S_{1,1}\right)
$$

$$
\sum_{m=1}^{\infty} g_{m}\left(Q_{m}\right), \quad \sum_{m=1}^{\infty} g_{m}\left(Q_{m} \cdot R_{1,1}\right), \quad \text { and } \sum_{m=1}^{\infty} g_{m}\left(Q_{m} \cdot S_{1,1}\right) .
$$

Consequently,

and

$$
\overline{\overline{\sum_{m=1}^{\infty}\left(Q_{m} \cdot R_{1,1}\right)}}=\overline{\overline{\sum_{m=1}^{\infty} g_{m}\left(Q_{m} \cdot R_{1,1}\right)}}
$$

$$
\overline{\overline{\sum_{m=1}^{\infty}\left(Q_{m} \cdot S_{1,1}\right)}}=\overline{\overline{\sum_{m=1}^{\infty} g_{m}\left(Q_{m} \cdot S_{1,1}\right)} .}
$$

We clearly have, in view of (17),

$$
R_{2,1} \subset P_{2,1} \subset \sum_{m=1}^{\infty} g_{m}\left(Q_{m}\right)+\prod_{m=1}^{\infty}\left(P_{2,1}-\sum_{i=1}^{m-1} g_{i}\left(Q_{i}\right)\right)
$$

and also, by (16) and (17),

$$
R_{2,1} \subset R \subset \sum_{m=1}^{\infty} g_{m}\left(P_{1,1}-\sum_{i=1}^{m-1} Q_{i}\right)
$$

By (29) and (30),

$$
R_{2,1} \subset \sum_{m=1}^{\infty} g_{m}\left(Q_{m}\right)+\sum_{m=1}^{\infty}\left[\left(P_{2,1}-\sum_{i=1}^{m-1} g_{i}\left(Q_{i}\right)\right) \cdot g_{m}\left(P_{1,1}-\sum_{i=1}^{m-1} Q_{i}\right)\right],
$$

and hence, by (26),

$$
R_{2,1} \subset \sum_{m+1}^{\infty} g_{m}\left(Q_{m}\right)
$$

From this formula, with the help of (9), (17), and (21), we obtain

$$
R_{2,1} \subset \sum_{m=1}^{\infty}\left(g_{m}\left(Q_{m}\right) \cdot R\right)=\sum_{m=1}^{\infty}\left(g_{m}\left(Q_{m}\right) \cdot g_{m}(R)\right)=\sum_{m=1}^{\infty} g_{m}\left(Q_{m} \cdot R\right)
$$

Since, by (17) and (25),

we get from (31)

$$
Q_{m} \cdot R=Q_{m} \cdot P_{1,1} \cdot R=Q_{m t} \cdot R_{1,1}
$$

$$
R_{2,1} \subset \sum_{m=1}^{\infty} g_{m}\left(Q_{m} \cdot R_{1,1}\right)
$$

By comparing (27) with (32) we see that the power of the set $R_{2,1}$ is at most equal to that of the set $\sum_{m=1}^{\infty}\left(Q_{m} \cdot R_{1,1}\right)$, which is a subset of $R_{1,1}$. Hence, by (18),

$$
\mathrm{r}_{1} \geqslant \mathrm{r}_{2} .
$$

On the other hand, we conclude from (16) and (17) that

$$
S_{1,1} \subset P_{1,1}-\sum_{n=1}^{\infty} g_{n}\left(P_{1,1}-\sum_{m=1}^{\infty} Q_{m}\right) \text {. }
$$

By (6), (7), and (11), one of the functions $g_{n}$ is the identity function $f_{1,1}$ with $D\left(f_{1,1}\right)=P_{1,1}$. Therefore (33) implies

and hence

$$
S_{1,1} \subset P_{1,1}-\left(P_{1,1}-\sum_{m=1}^{\infty} Q_{m}\right) \subset \sum_{m=1}^{\infty} Q_{m},
$$

$$
S_{1,1}=\sum_{m=1}^{\infty}\left(Q_{m} \cdot S_{1,1}\right)
$$


We also have by (17), (22), and (26)

$$
\sum_{m=1}^{\infty} g_{m}\left(Q_{m} \cdot S_{1,1}\right) \subset \sum_{m=1}^{\infty}\left(g_{m}\left(Q_{m}\right) \cdot g_{m}(S)\right) \subset P_{2,1} \cdot S
$$

consequently, by (17),

$$
\sum_{m=1}^{\infty} g_{m}\left(Q_{m} \cdot \ddot{S}_{1,1}\right) \subset \dot{S}_{2,1}
$$

From (28), (34), and (35) we see that $S_{1,1}$ has the same power as a subset of $S_{2,1}$; therefore, by $(18)$,

$$
\mathfrak{s}_{1} \leqslant \mathfrak{s}_{2}
$$

By (20), (24), (33), and (36), the cardinals $\mathfrak{x}_{i}$ and $\mathfrak{s}_{t}$ satisfy all the conditions of the conclusion; and the proof is complete ${ }^{13}$ )

The result just obtained can be improved: the conclusions of Theorem 7 prove to hold if $m_{1}, \ldots, m_{k}$ and $n_{1}, \ldots . ., n_{k}$, are assumed. to be, not necessarily natural numbers, but powers of well ordered sets (i. e., either natural numbers or so-called alephs). The proof of this stronger theorem requires but a slight modification of the original argument and does not involve the axiom of choice 14 ).

We are now in a position to establish the fundamental theorems. of this paper.

13) The idea of this proof is related to the one which was applied in Sierpiński [2]. However, Sierpiński was dealing with a rather special situation; instead of the system of functions $f_{i, j}$ he had to consider only two function which, in addition, were assumed to coincide with their inverses; in consequence, some important points of his argument cannot be applied to more general situations. The difference is quite essential; we see no possibility of establishing the cancellation laws for multiples in the general ease by mechanically extending Sierpiński's proof for $m=2$ (cf. the remarks in Sierpiński [3], p. 153). Another, less essential difference between the two proofs consists in the fact that Sierpins ki considers and somehow classifies individual elements of the sets involved, while in the proof of Theorem 7 we make instead an" extensive use of algebra of sets and set-images.

14) Theorem 7 can also be generalized in another direction: instead of finite sums, we can consider simple infinite sums (sums of infinite series) of cardinals. In this case the proof requires an application of the axiom of choice restricted to denumerable families of sets, and is "almost effective" in the sense of Tarski [1]. The problem whether Theorem 7 - in the original or in the generalized form - can be extended to arbitrary cardinal algebras still remains open, though its solution is very likely affirmative.
Theorem 8. If $m \neq 0$ and $m \cdot \mathfrak{p} \leqslant m \cdot q$, then $\mathfrak{p} \leqslant q$. Proof: We have by hypothesis

$$
m \cdot \mathrm{p}+\mathrm{t}=m \cdot \mathrm{q}
$$

for some cardinal $t$. We put in Theorem $7: k=3, m_{1}=n_{2}=m$, $m_{2}=n_{1}=n_{3}=0, m_{3}=1, \mathfrak{p}_{1}=\mathfrak{p}, \mathfrak{p}_{2}=\mathfrak{q}$, and $\mathfrak{p}_{3}=\mathrm{t}$. By (1), the hypothesis of Theorem 7 is then satisfied; we conclude that there are cardinals $\mathfrak{r}_{i}$ and $\mathfrak{s}_{i}(i=1,2,3)$ with the following properties:

$$
\begin{aligned}
& \text { By Theorem 6, formulas (3) imply: } \\
& \qquad \mathfrak{r}_{1}=\mathfrak{x}_{2} .
\end{aligned}
$$

The conclusion follows immediately from (2), (4), and (5).

Thoorem 9. If $m \neq 0$ and $m \cdot \mathfrak{p}=m \cdot \mathfrak{q}$, then $\mathfrak{p}=\mathfrak{q}$.

Proof: By Theorems 1 and 8 .

$\therefore$ As a further consequence of Theorem 8 we obtain:

If $m \cdot p<m \cdot q$, the $p<q(\text { and } m \neq 0)^{15}$ ).

This is the converse of the statement given above as an equivalent form of Theorem 6 .

By combining the results obtained in theorems and corollaries 3 , $4,5,8$, and 9 , we arrive at more general formulations given in the following three corollaries.

Corollary 10. If $m \neq 0$ and $k \cdot \pi+m \cdot p \leqslant(k+1) \cdot n+m \cdot q$, then $p \leqslant n+q$.

Proof: By Theorem 3 we obtain from the hypothesis:

Hence a fortiori

$$
m \cdot p \leqslant \pi+m \cdot q \text {. }
$$

$$
m \cdot \mathfrak{p} \leqslant m \cdot \mathfrak{n}+m \cdot \mathfrak{q}=m \cdot(\mathfrak{n}+\mathfrak{q})
$$

The conclusion now follows by Theorem 8.

Corollary 11. If $m \neq 0$ and $k \cdot n+m \cdot \mathfrak{p} \leqslant k \cdot \pi+m \cdot q$, then $n+p \leqslant n+q$.

Proof: By Corollary 4 and Theorem 8 (arguing as in the preceding proof);

.... 15) Sée Lindenbaum-Tarski [1], p. 305 (a result of Lindenbaum). 
Corollary 12. If $m \neq 0$ and $k \cdot \mathfrak{n}+m \cdot \mathfrak{p}=k \cdot \mathfrak{n}+m \cdot \mathfrak{q}$, then $\mathfrak{n}+\mathrm{p}=\mathrm{n}+\mathrm{q}$.

Proof: By Theorem 1 and Corollary 11, or else by Corollary 5 and Theorem 9 .

In conclusion, I should like to discuss another interesting result of the arithmetic of cardinal numbers closely related to those previously established in this paper - in fact, the so-called Euclid's theorem for cardinals. This theorem was also found and published without proof by Lindenbaum. The proof which will be given here is based upon Theorems 3 and 8 , and has a purely arithmetical character. I do not recall the original proof of Lindenbaum and have no idea to what extent, if any, the proof outlined below is related to it $\left.{ }^{16}\right)$.

Theorem 13. If the natural numbers $m$ and $n$ are relatively prime and $m \cdot \mathfrak{p}=n \cdot \mathfrak{q}$, then there is a cardinal number, $\mathfrak{x}$ such that $\mathfrak{p}=n \cdot \mathfrak{r}$ and $\mathfrak{q}=m \cdot \mathfrak{r}$.

Proof: We proceed by induction with respect to $\max (m, n)$. The case $\max (m, n)=0$ being impossible, and the case $\max (m, n)=1$ being obvious, we assume that $\max (m, n)>1$. We also assume that the conclusion of the theorem holds for all couples of relatively prime numbers $m^{\prime}$ and $n^{\prime}$ and for all couples of cardinals $p^{\prime}$ and $q^{\prime}$ such that

$$
\max \left(m^{\prime}, n^{\prime}\right)<\max (m, n) \text { and } m^{\prime} \cdot \mathfrak{p}^{\prime}=n^{\prime} \cdot \mathfrak{q}^{\prime} .
$$

One of the numbers $m$ and $n$ must be smaller than the other, say, $m<n$. We then have by hypothesis

hence by Theorem 8

$$
m \cdot \mathfrak{q} \leqslant m \cdot \mathfrak{p}(=n \cdot \mathfrak{q}) ;
$$

$$
\mathfrak{q} \leqslant \mathfrak{p}
$$

and therefore, for some cardinal $\mathfrak{s}$,

$$
q+\mathfrak{s}=\mathfrak{p} \text {. }
$$

Consequently, in view of the hypothesis,

(2)

and hence

$$
m \cdot q+m \cdot s=m \cdot p=n \cdot q,
$$

$$
m \cdot \mathfrak{q}+m \cdot \mathfrak{s} \leqslant(m+1) \cdot \mathfrak{q}+(n-m-1) \cdot \mathfrak{q} .
$$

28) The result in question is stated in Lindenbaum-Tarski [1], p. 305 . Another version of the proof given in the text can be found in Tarski [2], pp. 34f.
Therefore, by Theorem 3,

$$
m \cdot s \leqslant(n-m) \cdot q
$$

i. e., there exists a cardinal $t$ such that

(3)

This implies

$$
m \cdot \mathfrak{s}+\mathrm{t}=(n-m) \cdot \mathrm{q} \text {. }
$$$$
m \cdot q+m \cdot s+t=n \cdot q .
$$

By comparing (2) and (4) we obtain

hence, by Corollary 5,

$$
n \cdot \mathfrak{q}+\mathrm{t}=n \cdot \mathfrak{q}
$$

$$
q+t=q .
$$

From (5) we get (with the help of an easy induction)

$$
(n-m) \cdot \mathfrak{q}=(n-m) \cdot \mathfrak{q}+\mathrm{t}=(n-m) \cdot \mathfrak{q}+(m-1) \cdot \mathrm{t} ;
$$

together with (3), this gives

$$
(n-m) \cdot q=m \cdot(\mathfrak{s}+\mathfrak{t}) .
$$

Clearly, the numbers $n-m$ and $m$ are relatively prime, and

$$
\max (n-m, m)<\max (m, n)
$$

(since $m$ cannot equal 0 ); hence, by out inductive premise, (6) implies the existence of a cardinal $\mathfrak{r}$ for which

and

$$
q=m \cdot \mathfrak{r}
$$

$$
\mathfrak{s}+\mathrm{t}=(n-m) \cdot \mathfrak{r} .
$$

Formulas (1) and (5) give

$$
p=q+(s+t)
$$

consequently, by (7) and (8).

$$
p=n \cdot r .
$$

In view of (7) and (9), the cardinal $\mathfrak{r}$ satisfies the conditions of the conclusion. Hence the theorem holds for any natural numbers $m$ and $n$. 
Corollary 14. If $m$ and $n$ are any two natural numbers, not both zero, having $d$ as their greatest common divisor, and if $m \cdot \mathfrak{p}=n \cdot \mathfrak{q}$, then there is a cardinal $\mathfrak{r}$ such that $\mathfrak{p}=\frac{n}{d} \cdot \mathfrak{r}$ and $q=\frac{m}{d} \cdot x$.

Proof: By Theorem 9 (with $m=d$ ), the hypothesis implies

$$
\frac{m}{d} \cdot p=\frac{n}{d} \cdot q
$$

Hence, the natural numbers $\frac{m}{d}$ and $\frac{n}{d}$ being relatively prime, the conclusion follows by Theorem 13 ."

Corollary 14 clearly comprehends both Theorem 9 and Theorem 13 as particular cases." "

\section{Bibliography.} (1905-6).

Bernstein F. [1] Untersuchungen aus der. Mengentehre. Math. Ann. 61

Lindenbaum A. and Tarski A. [1] Oommunication sur les recherches de la théorie des ensembles. Comptes rendus des séances de la Société des Sciences et des Lettres de V Varsovie, Classe III, 19 (1926).

Sierpiński W. [1] Legons sur les nombres transfinis. Collection de monographies sur la théorie des fonctions. Paris 1928. (1922):

- [2] Sur l'égalité $2 \mathfrak{m}=2 \mathfrak{n}$ pour les nombres cardinaux. Fund. Math. 3.

- [3] Sur l'implication $(2 \mathfrak{m} \leqslant 2 \mathfrak{n}) \rightarrow(\mathfrak{m} \leqslant \mathfrak{n})$ pour les nombres cardinaux. Fund. Math. 34 (1946), pp. 148-154.

Tarski A. [1] Axiomatic and algebraic aspects of two theorems on sums of cardinals. Fund. Math. 35 (1948).

- [2] Cardinal algebras. New York, 1949.

\section{Retraction properties for normal Hausdorff spaces $^{1}$.}

By

\section{W. Saalfrank (Lancaster, Pennsylvania U.S.A.).}

1. Introduction. The idea of a retract was formulated by K. Borsuk [1] and retraction properties for separable metric spaces were developed by K. Borsuk, N. Aronszajn [3], and others. This theory has played a prominent role in investigations concerned with separable metric spaces. It is the purpose of this paper to develop a similar theory for normal Hausdorff spaces.

Section two will be devoted to results of a preliminary nature. In the next section we give definitions of absolute retract and absolute neighborhood retract for normal Hausdorff spaces. These concepts are characterized with the aid of the Tychonoff cube. We show in section four that the topological product of any set of absolute retracts or any finite set of absolute neighborhood retracts is an absolute retract or an absolute neighborhood retract, respectively. In the next section, under certain conditions, we prove that the union of two absolute retracts or absolute neighborhood retracts is again an absolute retract or an absolute neighborhood retract, respectively, in a restricted sense.

Section six is concerned with an extension of Borsuk's wellknown theorem [4, p. 86] on the extension of continuous maps into a $n$-sphere. Borsuk's theorem states: If $C$ is a closed subset of a separable metric space $X$, then for any continuous map $f:(X \times 0) \cup(C \times(0,1)) \rightarrow N$, where $N$ is a $n$-sphere or more generally a separable metric absolute neighborhood retract, there exists an extension $F$ of $f$ over $X \times(0,1)$, such that $F: X \times(0,1) \rightarrow N$. We prove this theorem for a compact Hausdorff space $X$, substituting a retract $B$ of any absolute retract $A$ for the point 0 and the unit

1) A Dissertation accepted by the University of Pennsylvania in fulfillment of the research requirement for the Degree of Doctor of Philosophy. 